\title{
THE STRUCTURE OF THE REVERSE HÖLDER CLASSES
}

\author{
DAVID CRUZ-URIBE, SFO AND C. J. NEUGEBAUER
}

\begin{abstract}
In this paper we study the structure of the class of functions $\left(R H_{s}\right)$ which satisfy the reverse Hölder inequality with exponent $s>1$. To do so we introduce a new operator, the minimal operator, which is analogous to the Hardy-Littlewood maximal operator, and a new class of functions, $\left(R H_{\infty}\right)$, which plays the same role for $\left(R H_{s}\right)$ that the class $\left(A_{1}\right)$ does for the $\left(A_{p}\right)$ classes.
\end{abstract}

\section{INTRODUCTION}

A non-negative, locally integrable function on $\mathbb{R}^{n}$ satisfies the reverse Hölder inequality with exponent $s>1$ if there exists a constant $C$ such that, for every cube $I$ in $\mathbb{R}^{n}$ with sides parallel to the co-ordinate axes,

$$
\left(\frac{1}{|I|} \int_{I} w^{s} d x\right)^{1 / s} \leq \frac{C}{|I|} \int_{I} w d x
$$

where $|I|$ denotes the Lebesgue measure of $I$. We say that such functions belong to the reverse Hölder class $\left(R H_{s}\right)$. Functions in these classes were first studied by Gehring [11] and by Coifman and C. Fefferman [7].

The $\left(R H_{s}\right)$ classes are closely related to the $\left(A_{p}\right)$ classes. A non-negative function $w$ is an $\left(A_{p}\right)$ weight, $p>1$, if

$$
\sup _{I}\left(\frac{1}{|I|} \int_{I} w d x\right)\left(\frac{1}{|I|} \int_{I} w^{1-p^{\prime}} d x\right)^{p-1}<\infty,
$$

where the supremum is taken over all cubes $I$ in $\mathbb{R}^{n}$ with sides parallel to the co-ordinate axes and $p^{\prime}$ is the conjugate exponent of $p$. A function $w$ is in $\left(A_{1}\right)$ if for some constant $C, M w \leq C w$, where $M$ is the Hardy-Littlewood maximal operator. The connection between the two classes is given by the following theorem [7]: a function $w$ is in $\left(A_{p}\right)$ for some $p>1$ if and only if it is in $\left(R H_{s}\right)$ for some $s>1$.

Much more is known about the $\left(A_{p}\right)$ classes than the $\left(R H_{s}\right)$ classes, and the purpose of this paper is to study the structure of the latter. Our main tool is a class of weights which play the same role for $\left(R H_{s}\right)$ weights that $\left(A_{1}\right)$ weights do for $\left(A_{p}\right)$ weights. To define this class we introduce a new operator,

Received by the editors July $11,1994$.

1991 Mathematics Subject Classification. Primary 42B25.

Key words and phrases. $\left(A_{p}\right)$ weights, reverse Hölder inequality, minimal function. 
the minimal operator, so named since it is analogous to the Hardy-Littlewood maximal operator.

Definition 1.1. If $f$ is a locally integrable function, define the minimal function of $f, m f$, by

$$
m f(x)=\inf _{I} \frac{1}{|I|} \int_{I}|f| d y,
$$

where the infimum is taken over all cubes $I$ with sides parallel to the co-ordinate axes which contain $x$.

It is immediate that $m f$ is a locally bounded function, and by the Lebesgue differentiation theorem $m f(x) \leq|f(x)|$ almost everywhere.

Using the minimal operator we define the class $\left(R H_{\infty}\right)$.

Definition 1.2. A non-negative function $w$ is an $\left(R H_{\infty}\right)$ weight if there exists a constant $C$ such that $w(x) \leq C m w(x)$ for almost every $x$.

This class was first defined by Franchi [9]; however, he defined it to be those functions $w$ such that for every cube $I, w(x) \leq C I(w)$ for almost every $x$ in $I$. An argument identical to that for $\left(A_{1}\right)$ weights (see [10, p. 389]) shows that these two definitions are equivalent. In [4], the first author gave a characterization of increasing $\left(A_{\infty}\right)$ weights on $\mathbb{R}_{+}$which shows that they are all in $\left(R H_{\infty}\right)$. Earlier, the $\left(R H_{\infty}\right)$ condition appeared in works of Andersen and Young [1] and Muckenhoupt [18]. (For further details on their work, see the appendix.)

The paper is organized as follows: we begin in Section 2 by stating some known results which relate the $\left(A_{p}\right)$ and $\left(R H_{s}\right)$ classes. They are included for completeness and ease of reference. As applications we examine the geometry of $\log \left(A_{p}\right)$ and $\log \left(R H_{s}\right)$ as subsets of $\mathrm{BMO}$, and we prove a two-weight version of the reverse Hölder inequality.

In Section 3 we digress slightly to prove two weighted norm inequalities for the minimal operator. These are included to develop the analogy between the minimal and maximal operators.

In Section 4 we examine $\left(R H_{\infty}\right)$ weights and show how they are related to $\left(A_{1}\right)$ weights. We conclude by determining the multipliers of $\left(R H_{\infty}\right)$; as a corollary we give a new proof of a characterization of the multipliers of $\left(A_{1}\right)$ discovered by Johnson and Neugebauer [15]. In Section 5 we apply these results to study the reverse Hölder classes. We extend the Jones' factorization theorem to $\left(R H_{s}\right)$ weights, and we examine the action of the maximal and minimal operators on the $\left(A_{p}\right)$ and $\left(R H_{s}\right)$ classes.

In Section 6 we further examine the nature of $\left(R H_{\infty}\right)$ weights. We extend a result of Coifman and Rochberg (see Theorem 6.1 below) by characterizing those functions $w$ such that $M w$ is in $\left(A_{1}\right)$. From this we derive a characterization of those $w$ such that $m w$ is in $\left(R H_{\infty}\right)$. We end this section by discussing the relationship between our results and the so-called higher integrability theorems of Gehring and others.

The final section is an application: in Section 7 we give a new proof that the maximal operator is bounded on BMO, a result originally proved by Bennett, DeVore, and Sharpley [2]. The proof is based on a lemma which shows that for $\left(A_{p}\right)$ weights the maximal operator and the logarithm essentially commute. 
The appendix is an erratum to a paper of the first author [5]. The error was discovered while preparing this paper; in the correction we give another application of the class $\left(R H_{\infty}\right)$.

\section{NOTATION AND PRELIMINARY RESULTS}

Throughout this paper all notation is standard or will be defined as needed. All cubes in $\mathbb{R}^{n}$ have their edges parallel to the co-ordinate axes. All functions are locally integrable. For a cube $I$ and a function $w$, let $|I|$ be the Lebesgue measure of $I$ and define $w(I)=\int_{I} w d x$ and $I(w)=w(I) /|I|$. Given a cube $I$ and $k>0$, let $k I$ be the cube with the same center whose edges are $k$ times as long. (Then $|k I|=k^{n}|I|$.)

Given two functions $f$ and $g$, they are equivalent if $f / g$ is bounded and bounded away from zero. This equivalence relation is denoted by $f \sim g$.

Finally, given $p>1, p^{\prime}=p /(p-1)$ is the conjugate exponent of $p$. The letter $C$ denotes a non-negative constant whose value may be different at each appearance.

Since $\left(R H_{s}\right)$ weights are in $\left(A_{\infty}\right)$, below we will use many of the properties of $\left(A_{\infty}\right)$ weights. We will refer frequently to the treatment given by GarciaCuerva and Rubio de Francia in [10]. The union of all the $\left(A_{p}\right)$ classes is denoted by $\left(A_{\infty}\right)$. In addition, we define $\left(A_{*}\right)$ to be intersection of all the $\left(A_{p}\right)$ classes, $p>1$, and $\left(R H_{*}\right)$ to be the intersection of all the $\left(R H_{s}\right)$ classes, $s>1$.

Our first result was discovered by Strömberg and Wheeden [22]. (A different proof is given in [15].) It is an invaluable tool in studying the reverse Hölder classes.

Theorem 2.1. A function $w$ is in $\left(R H_{s}\right), 1<s<\infty$, if and only if $w^{s}$ is in $\left(A_{\infty}\right)$.

This theorem is quantified by the following two results. The first follows by applying Hölder's inequality and then the reverse Hölder inequality in succession; a proof is found in [16]. A proof of the second is found in [15].

Theorem 2.2. A function $w$ is in $\left(A_{p}\right) \cap\left(R H_{s}\right)$ if and only if $w^{s}$ is in $\left(A_{q}\right)$, where $q=s(p-1)+1$.

Theorem 2.3. If $w$ is in $\left(A_{\infty}\right)$ and $w^{r}$ is in $\left(A_{1}\right)$ for some $r>0$, then $w$ is in $\left(A_{1}\right)$.

We will use all three of these results repeatedly below. As an immediate application we will use Theorem 2.1 to examine the geometry of the reverse Hölder classes. (Additional results are given in Corollary 4.7 below.)

Theorem 2.4. The sets

$$
\log \left(R H_{s}\right)=\left\{\phi \in \mathrm{BMO}: e^{\phi} \in\left(R H_{s}\right)\right\}, \quad 1<s<\infty,
$$

are open and convex in BMO. The set $\log \left(A_{*}\right)$ is neither open nor closed in $B M O$; however, the set $\log \left(R H_{*}\right)$ is closed.

Proof. By Theorem 2.1 the set $\log \left(R H_{s}\right)$ is just the contraction of the set $\log \left(A_{\infty}\right)$ by a factor of $s$. This latter set is open [17] and convex [15] in $\mathrm{BMO}$, so $\log \left(R H_{s}\right)$ is as well. 
To see that $\log \left(A_{*}\right)$ is not closed in BMO, note that in $\mathbb{R}^{n}$ the function $w(x)=|x|^{-r n}, 0<r<1$, is in $\left(A_{1}\right)$ and so in $\left(A_{*}\right)[10$, p. 160], but the limit function $w(x)=|x|^{-n}$ is not even locally integrable around the origin. Hence $-n \log |x|$ is a limit point of $\log \left(A_{*}\right)$ but is not contained in it.

To show that $\log \left(A_{*}\right)$ is not open, suppose to the contrary that it is. Since $w(x) \equiv 1$ is in $\left(A_{*}\right)$, there exists an $\epsilon>0$ such that if $\|\phi\|_{*}<\epsilon$ then $\phi$ is in $\log \left(A_{*}\right)$. Fix the dimension as $n$, and let $\phi(x)=(p-1) n \log |x|$. Then for some $p$ sufficiently close to one, $\phi$ is in $\log \left(A_{*}\right)$; in particular it is in $\log \left(A_{p}\right)$. Hence, by the duality of $\left(A_{p}\right)$ weights, $|x|^{-n}=e^{\phi\left(1-p^{\prime}\right)}$ is in $\left(A_{p^{\prime}}\right)$, a contradiction.

Finally, that $\log \left(R H_{*}\right)$ is closed follows from the fact that functions in $\left(R H_{*}\right)$ are exactly the multipliers of $\left(A_{\infty}\right)$ [15]. Let $\phi$ be a limit point of $\log \left(R H_{*}\right)$; it will suffice to show that $\phi+\psi$ is in $\log \left(A_{\infty}\right)$ whenever $\psi$ is. Since $\log \left(A_{\infty}\right)$ is open, for each such $\psi$ there exists $\epsilon>0$ such that the ball, $B$, of radius $\epsilon$ around $\psi$ is contained in $\log \left(A_{\infty}\right)$. Since $\phi$ is a limit point of $\log \left(R H_{*}\right)$, there exists $\phi_{0}$ in $\log \left(R H_{*}\right)$ such that $\left\|\phi-\phi_{0}\right\|<\epsilon$. Since $e^{\phi_{0}}$ is a multiplier of $\left(A_{\infty}\right), B+\phi_{0}$ is contained in $\log \left(A_{\infty}\right)$. But $\phi+\psi$ is in $B+\phi_{0}$, so we are done.

We end this section with a two-weight version of the reverse Hölder inequality. Originally we derived it as a lemma for the proof of Theorem 2.4; even though that proof was simplified, we retain this result since it is of independent interest. (Also, an application of it is given in Theorem 4.8 below.)

Lemma 2.5. Fix $p \geq 2$. If $w$ is in $\left(A_{p}\right)$ and $v$ is in $\left(A_{p^{\prime}}\right)$, then $w^{1 / p} v^{1 / p^{\prime}}$ is in $\left(A_{p}\right)$ and there exists a constant $C$ such that for every cube $I$

$$
I(w)^{1 / p} I(v)^{1 / p^{\prime}} \leq C I\left(w^{1 / p} v^{1 / p^{\prime}}\right) .
$$

Proof. Since $p \geq 2, p^{\prime} \leq p$. Therefore both $v$ and $w$ are in $\left(A_{p}\right)$; since $\log \left(A_{p}\right)$ is convex [15], $w^{1 / p} v^{1 / p^{\prime}}$ is also in $\left(A_{p}\right)$.

To establish inequality (1), fix a cube $I$. Then there is a constant $C$ such that

$$
I(w) I\left(w^{1-p^{\prime}}\right)^{p-1} \leq C, \quad I(v) I\left(v^{1-p}\right)^{p^{\prime}-1} \leq C .
$$

Raise the first equation to the power $1 / p$ and the second to the power $1 / p^{\prime}$ and multiply them together. Then after re-arranging terms we get

$$
I(w)^{1 / p} I(v)^{1 / p^{\prime}} \leq C I\left(w^{1-p^{\prime}}\right)^{-1 / p^{\prime}} I\left(v^{1-p}\right)^{-1 / p} .
$$

Since $p / p^{\prime}=p-1$ and $p^{\prime} / p=p^{\prime}-1$, by Hölder's inequality

$$
I\left(w^{-1 / p} v^{-1 / p^{\prime}}\right) \leq I\left(w^{1-p^{\prime}}\right)^{1 / p^{\prime}} I\left(v^{1-p}\right)^{1 / p} .
$$

Therefore, by combining these two inequalities we see that

$$
I(w)^{1 / p} I(v)^{1 / p^{\prime}} \leq C I\left(w^{-1 / p} v^{-1 / p^{\prime}}\right)^{-1}
$$

Now apply Hölder's inequality twice (it is possible the second time since $p \geq 2$ ):

$$
\begin{aligned}
I\left(w^{-1 / p} v^{-1 / p^{\prime}}\right)^{-1} & \leq I\left(\left(w^{-1 / p} v^{-1 / p^{\prime}}\right)^{1-p^{\prime}}\right)^{p-1} \\
& =I\left(\left(w^{1 / p} v^{1 / p^{\prime}}\right)^{p^{\prime}-1}\right)^{p-1} \\
& \leq I\left(w^{1 / p} v^{1 / p^{\prime}}\right) .
\end{aligned}
$$

Therefore inequality (1) holds. 
The desired version of this result is stated without reference to $\left(A_{p}\right)$ classes.

Theorem 2.6. Fix $p>1$. If $w$ is in $\left(R H_{p}\right)$ and $v$ is in $\left(R H_{p^{\prime}}\right)$, then there exists a constant $C$ such that for every cube $I$

$$
\left(\int_{I} w^{p} d x\right)^{1 / p}\left(\int_{I} v^{p^{\prime}} d x\right)^{1 / p^{\prime}} \leq C \int_{I} w v d x
$$

Proof. By Theorems 2.1 and 2.2, there exists $s<1$ such that $w^{s p}$ is in $\left(A_{p}\right) \cap$ $\left(R H_{1 / s}\right)$ and $v^{s p^{\prime}}$ is in $\left(A_{p^{\prime}}\right) \cap\left(R H_{1 / s}\right)$. Therefore, by Lemma 2.5,

$$
\begin{aligned}
I\left(w^{p}\right)^{s / p} I\left(v^{p^{\prime}}\right)^{s / p^{\prime}} & \leq C I\left(w^{s p}\right)^{1 / p} I\left(v^{s p^{\prime}}\right)^{1 / p^{\prime}} \\
& \leq C I\left(w^{s} v^{s}\right) \\
& \leq C I(w v)^{s} .
\end{aligned}
$$

If we raise both sides to the power $1 / s$ and multiply by $|I|$ we get inequality (2).

It is an open question to characterize pairs of weights $w$ and $v$ which satisfy this inequality. Here we give one partial result.

Theorem 2.7. Suppose $w$ and $v$ satisfy inequality (2) for some $p>1$ and $w$ is in $\left(R H_{p}\right)$. Then $v$ is in $\left(R H_{p^{\prime}}\right)$.

Proof. By Theorem 2.1, $w^{p}$ is in $\left(A_{\infty}\right)$ and so in $\left(R H_{r / p}\right)$ for some $r>p$. Now apply Hölder's inequality to inequality (2):

$$
\begin{aligned}
I\left(w^{p}\right)^{1 / p} I\left(v^{p^{\prime}}\right)^{1 / p^{\prime}} & \leq C I(w v) \\
& \leq C I\left(w^{r}\right)^{1 / r} I\left(v^{r^{\prime}}\right)^{1 / r^{\prime}} \\
& \leq C I\left(w^{p}\right)^{1 / p} I\left(v^{r^{\prime}}\right)^{1 / r^{\prime}}
\end{aligned}
$$

Since $r>p, r^{\prime}<p^{\prime}$. Therefore, $v^{r^{\prime}}$ is in $\left(R H_{p^{\prime} / r^{\prime}}\right)$, so, again by Theorem $2.1, v$ is in $\left(R H_{p^{\prime}}\right)$.

\section{THE MINIMAL OPERATOR}

In this section we prove two weighted norm inequalities for the minimal operator. To do so, we need to identify the "natural domain" of the minimal operator. For the maximal operator, the natural domains are the $L^{p}$ spaces, $p>1$, since, intuitively, the maximal operator "controls" the behavior of a function where it is large. But if $f$ is in any $L^{p}$ space, $p<\infty$, then $m f \equiv 0$.

The minimal operator controls the behavior of a function $f$ where it is small, and any norm inequality needs to reflect this fact. We do this in Theorems 3.1 and 3.4 by replacing $f$ by $1 / f$ and $\log f$, which are large where $f$ is small. Thus the natural domains of the minimal operator consist of those functions $f$ for which $1 / f$ or $\log f$ are integrable. 
Theorem 3.1. Given a function $w$, a necessary and sufficient condition for the weighted norm inequality

$$
\int_{\mathbb{R}^{n}} \frac{w}{(m f)^{p}} d x \leq C \int_{\mathbb{R}^{n}} \frac{w}{|f|^{p}} d x
$$

to hold for all $p>0$ and all $f$ such that $1 / f$ is in $L^{p}(w)$ is for $w$ to be in $\left(A_{\infty}\right)$. The constant $C$ depends only on $w$ and is independent of $p$.

Proof. Suppose first that $w$ is in $\left(A_{\infty}\right)$. Then $w$ is in $\left(A_{q}\right)$ for some $q>1$, and the maximal function is a bounded operator on $L^{q}(w)$ [10, p. 400]. Fix $p>0$ and take $r>1$ such that $(r-1) p=q$. Let $f$ be any function such that $1 / f$ is in $L^{p}(w)$. By Hölder's inequality, $m f(x)^{-1} \leq M\left(f^{1-r^{\prime}}\right)(x)^{r-1}$. Hence there exists a constant $C$ depending only on the $\left(\bar{A}_{q}\right)$ constant of $w$ such that

$$
\int_{\mathbb{R}^{n}} \frac{w}{(m f)^{p}} d x \leq \int_{\mathbb{R}^{n}} M\left(f^{1-r^{\prime}}\right)^{(r-1) p} w d x \leq C \int_{\mathbb{R}^{n}} \frac{w}{|f|^{p}} d x,
$$

which is exactly inequality (3).

Conversely, suppose $w$ is such that inequality (3) holds for all $p>0$. Fix a cube $I$ and define the function $f(x)=w(x)^{\alpha} / \chi_{I}(x)$, where $\alpha=1 /(1+p)$. For all $x$ in $I, m f(x) \leq I\left(w^{\alpha}\right)$; so inequality (3) implies that

$$
\frac{w(I)}{I\left(w^{\alpha}\right)^{p}} \leq C \int_{I} w^{1-\alpha p} d x
$$

Since $\alpha=1-\alpha p$, inequality (4) becomes

$$
I(w) \leq C\left(\frac{1}{|I|} \int_{I} w^{1 /(p+1)} d x\right)^{p+1}
$$

The constant $C$ is independent of $I$. Hence $w^{1 /(p+1)}$ is in $\left(R H_{p+1}\right)$, and so by Theorem $2.1 w$ is in $\left(A_{\infty}\right)$.

Theorem 3.1 has the following corollary, which we give for comparison with Theorem 3.4 below.

Corollary 3.2. Let the function $f$ be such that $f(x) \geq 1$. Then for $w$ in $\left(A_{\infty}\right)$

$$
\int_{\mathbb{R}^{n}} \frac{w}{\log m f} d x \leq C \int_{\mathbb{R}^{n}} \frac{w}{\log f} d x,
$$

where the constant $C$ depends only on $w$.

Proof. Since $w$ is in $\left(A_{\infty}\right)$, inequality (3) holds for all $p>0$ with a constant independent of $p$. Integrate both sides of this inequality with respect to $p$. Then

$$
\int_{0}^{\infty} \int_{\mathbb{R}^{n}} \frac{w}{(m f)^{p}} d x d p \leq C \int_{0}^{\infty} \int_{\mathbb{R}^{n}} \frac{w}{f^{p}} d x d p
$$

Apply Fubini's theorem, and inequality (5) follows at once.

Question 3.3. Does inequality (5) imply that $w$ is in $\left(A_{\infty}\right)$ ? 
Theorem 3.4. Given a function $w$, a necessary and sufficient condition for the weighted-norm inequality

$$
\int_{\mathbb{R}^{n}}|\log m f|^{p} w d x \leq C \int_{\mathbb{R}^{n}}|\log f|^{p} w d x,
$$

to hold for $p>1$ and all $f$ such that $0 \leq f(x) \leq 1$ and $\log f$ is in $L^{p}(w)$, is for $w$ to be in $\left(A_{p}\right)$. The constant $C$ depends only on the $\left(A_{p}\right)$ constant of $w$.

Proof. Suppose first that $w$ is in $\left(A_{p}\right)$. Let $f$ be as in the hypotheses. Then by Jensen's inequality, at each point $x$

$$
\log (\inf I(f))=\inf (\log I(f)) \geq \inf I(\log f),
$$

where each infimum is taken over all cubes $I$ which contain $x$. Since $f$ is less than one, $m f(x) \leq 1$. Therefore,

$$
|\log m f(x)| \leq|\inf I(\log f)|=\sup I(|\log f|)=M(\log f)(x) .
$$

Inequality (6) follows immediately from this: since $w$ is in $\left(A_{p}\right)$, the maximal operator is bounded on $L^{p}(w)$, so

$$
\int_{\mathbb{R}^{n}}|\log m f|^{p} w d x \leq \int_{\mathbb{R}^{n}} M(\log f)^{p} w d x \leq C \int_{\mathbb{R}^{n}}|\log f|^{p} w d x .
$$

The constant $C$ depends only on the $\left(A_{p}\right)$ constant of $w$.

To prove the converse, suppose $w$ is such that (6) holds for all $f$. Fix $a$, $0<a<1$. On $(0,1)$ define the function $\Phi_{a}(t)=\log (1 / t)^{a}$. Then a simple calculation shows that $\Phi_{a}$ is monotonic and concave on $\left(e^{a-1}, 1\right)$. Now let $f$ be any function such that $\epsilon \leq f(x) \leq 1$ for some $\epsilon>0$. Then there exists $k>0$ such that $f(x)^{k}>e^{a-1}$. Therefore, by Jensen's inequality, for any cube $I, I\left(\Phi_{a}\left(f^{k}\right)\right) \leq \Phi_{a}\left(I\left(f^{k}\right)\right)$, or equivalently,

$$
I\left(\left|\log \left(f^{k}\right)\right|^{a}\right)^{1 / a} \leq\left|\log I\left(f^{k}\right)\right| .
$$

Fix $x$ and take the supremum over all $I$ containing $x$. Then

$$
M\left(\left|\log \left(f^{k}\right)\right|^{a}\right)^{1 / a} \leq\left|\log m\left(f^{k}\right)\right| .
$$

Therefore, by inequality (6) we see that

$$
\int_{\mathbb{R}^{n}} M\left(|\log f|^{a}\right)^{p / a} w d x \leq C \int_{\mathbb{R}^{n}}|\log f|^{p} w d x ;
$$

we could eliminate the $k$ 's since the maximal operator is positive homogeneous.

Since bounded functions are dense in $L^{p / a}(w)$, it follows from inequality (7) that the maximal operator is bounded on $L^{p / a}(w)$. Therefore $w$ is in $\left(A_{p / a}\right)$ $\left[10\right.$, p. 400]. However, the constant in (7) is independent of $a$, so the $\left(A_{p / a}\right)$ constant of $w$ is as well. Hence $w$ is in $\left(A_{p}\right)$.

As a corollary to Theorem 3.4 we get the following commutation result. It should be compared with Theorem 7.1 below. 
Corollary 3.5. Let $w$ be in $\left(A_{p}\right), p>1$. Then, for every function $f$ such that $0 \leq f(x) \leq 1$ and $\log f$ is in $L^{p}(w)$,

$$
\int_{\mathbb{R}^{n}} M(|\log f|)^{p} w d x \sim \int_{\mathbb{R}^{n}}|\log m f|^{p} w d x .
$$

Though we will not discuss them here, the minimal operator has other applications to weighted norm inequalities and to differentiation theory. These results will appear in [6].

\section{THE CLASS $\left(R H_{\infty}\right)$}

In this section we examine the properties of $\left(R H_{\infty}\right)$ weights. We begin by showing that they have a number of properties similar to those of $\left(A_{1}\right)$ weights.

It follows at once from Definition 1.2 that just as $\left(A_{1}\right)$ weights are locally bounded away from zero, $\left(R H_{\infty}\right)$ weights are locally bounded.

The class $\left(A_{1}\right)$ is a proper subset of $\left(A_{*}\right)$; similarly, $\left(R H_{\infty}\right)$ is a proper subset of $\left(R H_{*}\right)$. We show inclusion in Theorem 4.1 and give an example afterward to show that the inclusion is proper.

Theorem 4.1. If $w$ is in $\left(R H_{\infty}\right)$, then $w$ is in $\left(R H_{*}\right)$ and for each $s>1$ the $\left(R H_{s}\right)$ constant of $w$ is bounded by the $\left(R H_{\infty}\right)$ constant of $w$. Conversely, if $w$ is in $\left(R H_{*}\right)$ and for each $s>1$ its $\left(R H_{s}\right)$ constant is independent of $s$, then $w$ is in $\left(R H_{\infty}\right)$.

Proof. Suppose that $w$ is in $\left(R H_{\infty}\right)$. Fix a cube $I$; then for almost every $x$ in $I, C_{0} I(w) \geq w(x)$, where $C_{0}$ is the $\left(R H_{\infty}\right)$ constant of $w$. Fix $s>1$, raise both sides of this inequality to the $s$-th power and integrate with respect to $x$ over $I$. Then $C_{0}^{s} I(w)^{s} \geq I\left(w^{s}\right)$. Hence $w$ is in $\left(R H_{s}\right)$ and its $\left(R H_{s}\right)$ constant is bounded by its $\left(R H_{\infty}\right)$ constant.

Conversely, suppose that $w$ is in $\left(R H_{*}\right)$ and there is a constant $C_{1}$ such that for each $s>1, I\left(w^{s}\right)^{1 / s} \leq C_{1} I(w)$ for every cube $I$. Take the limit as $s$ tends to infinity. The left-hand side tends to the essential supremum of $w$ on $I$, so $w(x) \leq C_{1} I(w)$ for almost every $x$ in $I$. Hence $w$ is $\left(R H_{\infty}\right)$.

To see that the inclusion of $\left(R H_{\infty}\right)$ in $\left(R H_{*}\right)$ is proper, consider the function $w(x)=\max \{\log (1 /|x|), 1\}$. A calculation shows that it in $\left(R H_{s}\right), s>1$; however, it is unbounded in any neighborhood of the origin and so cannot be in $\left(R H_{\infty}\right)$.

The analogue of the second half of Theorem 4.1 for $\left(A_{1}\right)$ weights does not appear to be stated explicitly in the literature, but it is implicit in any description of the $\left(A_{1}\right)$ condition as the limit of the $\left(A_{p}\right)$ condition as $p$ tends to one. For example, see [10, p. 391].

By Theorem 2.3, if $w$ is in $\left(A_{1}\right) \cap\left(R H_{s}\right)$, then $w^{r}$ is in $\left(A_{1}\right)$ for every $r, 0<r \leq s$. A much stronger result holds for functions in $\left(R H_{\infty}\right)$ :

Theorem 4.2. Suppose $w$ is in $\left(R H_{\infty}\right)$. Then for any $r>0, w^{r}$ is also in $\left(R H_{\infty}\right)$.

Proof. We treat two cases. If $r>1$, then by Hölder's inequality $I\left(w^{r}\right) \geq I(w)^{r}$ for every cube $I$, so $w^{r}$ in $\left(R H_{\infty}\right)$ follows at once from the fact that $w$ is in $\left(R H_{\infty}\right)$. 
If $r<1$, then by Theorem $2.1 w^{r}$ is in $\left(R H_{1 / r}\right)$. So given a cube $I$, for almost every $x$ in $I, w(x)^{r} \leq I(w)^{r} \leq C I\left(w^{r}\right)$. Hence $w^{r}$ is in $\left(R H_{\infty}\right)$.

These similarities between the classes $\left(A_{1}\right)$ and $\left(R H_{\infty}\right)$ are due to a duality between them which can be roughly described as: a function $w$ is in $\left(A_{1}\right)$ if and only if $1 / w$ is in $\left(R H_{\infty}\right)$. This statement is not completely accurate, since the relationship is not completely symmetric. Before we can describe the relationship exactly, we need to prove the following lemma.

Lemma 4.3. If $w$ is in $\left(A_{p}\right), p>1$, then there exists a constant $C$ such that

$$
1 \leq m w M\left(w^{1-p^{\prime}}\right)^{p-1} \leq C
$$

and

$$
1 \leq M w m\left(w^{1-p^{\prime}}\right)^{p-1} \leq C .
$$

Proof. We will prove inequality (8); the proof of inequality (9) is identical.

Fix $x$ and let $I$ be any cube containing $x$. Since $w$ is in $\left(A_{p}\right)$, there exists a constant $C$ such that

$$
I(w)^{-1} \leq I\left(w^{1-p^{\prime}}\right)^{p-1} \leq C I(w)^{-1} .
$$

Take the supremum over all cubes $I$ containing $x$. Since $\sup I(w)^{-1}=$ $(\inf I(w))^{-1}$, we get

$$
m w(x)^{-1} \leq M\left(w^{1-p^{\prime}}\right)(x)^{p-1} \leq C m w(x)^{-1},
$$

which is inequality (8).

Theorem 4.4. Fix $p>1$. If $w$ is in $\left(A_{1}\right)$, then $w^{1-p}$ is in $\left(R H_{\infty}\right) \cap\left(A_{p}\right)$. Conversely, if $w$ is in $\left(R H_{\infty}\right) \cap\left(A_{p}\right)$, then $w^{1-p^{\prime}}$ is in $\left(A_{1}\right)$.

Proof. If $w$ is in $\left(A_{1}\right)$ it is in $\left(A_{p^{\prime}}\right)$; hence, by the duality of $\left(A_{p}\right)$ weights, $w^{1-p}$ is in $\left(A_{p}\right)$. To see that it is in $\left(R H_{\infty}\right)$, note that by inequality (9)

$$
1 \leq M w(x) m\left(w^{1-p}\right)(x)^{p^{\prime}-1} \leq C w(x) m\left(w^{1-p}\right)(x)^{p^{\prime}-1} ;
$$

the last inequality holds almost everywhere since $w$ is in $\left(A_{1}\right)$. By re-arranging this inequality we see that $w(x)^{1-p} \leq C m\left(w^{1-p}\right)(x)$, so $w^{1-p}$ is in $\left(R H_{\infty}\right)$.

Now suppose that $w$ is in $\left(R H_{\infty}\right) \cap\left(A_{p}\right)$. Then by inequality (8),

$$
M\left(w^{1-p^{\prime}}\right)(x) \leq C m w(x)^{1-p^{\prime}} \leq C w(x)^{1-p^{\prime}}
$$

holds almost everywhere since $w$ is in $\left(R H_{\infty}\right)$. Hence $w^{1-p^{\prime}}$ is in $\left(A_{1}\right)$.

To see the lack of complete symmetry between $\left(A_{1}\right)$ and $\left(R H_{\infty}\right)$, note that by Theorem 4.4 if $w$ is in $\left(A_{1}\right)$, then $1 / w$ is in $\left(R H_{\infty}\right)$, but the converse is not true. A simple example of this in $\mathbb{R}$ is $w(t)=|t|$. Furthermore, even when $w^{1-p^{\prime}}$ is in $\left(A_{1}\right)$, we have no control over its reverse Hölder class: Given any $p>1$ and $s>1$, there exists a function $w$ in $\left(R H_{\infty}\right) \cap\left(A_{p}\right)$ such that $w^{1-p^{\prime}}$ is not in $\left(R H_{s}\right)$. For example, take $w(t)=|t|^{(p-1) / s}$.

Corollary 4.5 is more symmetric, but at the expense of unwanted complexity. 
Corollary 4.5. If $w$ is in $\left(A_{1}\right) \cap\left(R H_{s}\right)$ for all $s<s_{0}$, then for all $p>1$, $w^{1-p}$ is in $\left(R H_{\infty}\right) \cap\left(A_{q}\right)$ for all $q>(p-1) / s_{0}+1$. Conversely, if $w$ is in $\left(R H_{\infty}\right) \cap\left(A_{p}\right)$ for all $p>p_{0}$, then $w^{1-p^{\prime}}$ is in $\left(A_{1}\right) \cap\left(R H_{s}\right)$ for all $s<(p-1) /\left(p_{0}-1\right)$.

Proof. Suppose first that $w$ is in $\left(A_{1}\right) \cap\left(R H_{s}\right)$ for $s<s_{0}$. Then by Theorems 2.3 and 4.4, $\left(w^{s}\right)^{1-p}$ is in $\left(R H_{\infty}\right) \cap\left(A_{p}\right)$; therefore, by Theorems 2.2 and $4.2, w^{1-p}$ is in $\left(R H_{\infty}\right) \cap\left(A_{(p-1) / s+1}\right)$. Since this is true for all $s<s_{0}$, we obtain the desired bound.

Similarly, suppose $w$ is in $\left(R H_{\infty}\right) \cap\left(A_{p}\right)$ for all $p>p_{0}$. Fix $p$ and $p_{1}$, $p>p_{1}>p_{0}$. Then, again by Theorem $4.4, w^{1-p_{1}^{\prime}}=w^{\left(1-p^{\prime}\right) s}$ is in $\left(A_{1}\right)$, where

$$
s=\frac{1-p_{1}^{\prime}}{1-p^{\prime}}=\frac{p-1}{p_{1}-1} \text {. }
$$

Thus by Theorem $2.1, w^{1-p^{\prime}}$ is in $\left(R H_{s}\right)$. Since this is true for any $p_{1}>p_{0}$, the desired bound on $s$ follows at once.

To see the full symmetry, note that if $s_{0}=(p-1) /\left(p_{0}-1\right)$, then $p_{0}=$ $(p-1) / s_{0}+1$.

We give two more corollaries to Theorem 4.1. The first further characterizes $\left(R H_{\infty}\right)$ weights.

Corollary 4.6. A function $w$ is in $\left(R H_{\infty}\right)$ if and only if $\log (1 / w)$ is in $B L O$, the space of functions of bounded lower oscillation. In particular, if $w$ is in $\left(A_{1}\right) \cap\left(R H_{\infty}\right)$, then $w$ is bounded and bounded away from zero.

Proof. The first asssertion follows at once from Theorems 4.2 and 4.4 and the fact that the logarithm of an $\left(A_{1}\right)$ weight is in BLO [10, p. 409]. The second follows from the fact that if $\phi$ and $-\phi$ are both in BLO, then $\phi$ is a bounded function $[10$, p. 158].

The second corollary continues the description of the geometry of the sets $\log \left(A_{p}\right)$ and $\log \left(R H_{s}\right)$ begun in Theorem 2.4.

Corollary 4.7. The sets $\log \left(A_{1}\right)$ and $\log \left(R H_{\infty}\right)$ are convex in $B M O$ but are neither open nor closed.

Proof. That $\log \left(A_{1}\right)$ is convex follows immediately from Hölder's inequality. (See also [15].) By Theorem 4.4, $\log \left(R H_{\infty}\right) \cap \log \left(A_{p}\right)=(1-p) \log \left(A_{1}\right)$ and so is convex. Since this is true for all $p>1, \log \left(R H_{\infty}\right)$ is the union of nested convex sets and so convex.

The proof in Theorem 2.4 that $\log \left(A_{*}\right)$ is neither open nor closed also shows that $\log \left(A_{1}\right)$ is neither open nor closed. It follows from this that $\log \left(R H_{\infty}\right)$ cannot be open: for if it were, then $\log \left(R H_{\infty}\right) \cap \log \left(A_{p}\right)=(1-p) \log \left(A_{1}\right)$ would also be open since $\log \left(A_{p}\right)$ is open [17], a contradiction. Finally, to see that $\log \left(R H_{\infty}\right)$ is not closed, take any positive function $\phi$ in the BMO closure of $\mathbf{L}^{\infty}$ which is not bounded at the origin. Then $\phi$ is a limit point of $\log \left(R H_{\infty}\right)$, since this set contains $\mathrm{L}^{\infty}$, but is not contained in it.

We close this section by characterizing the multipliers of $\left(R H_{\infty}\right)$, that is, those functions $\phi$ such that $\phi w$ is in $\left(R H_{\infty}\right)$ whenever $w$ is.

Theorem 4.8. A function $\phi$ is a multiplier of $\left(R H_{\infty}\right)$ if and only if it is $\left(R H_{\infty}\right)$. 
Proof. If $\phi$ is a multiplier, then $\phi$ must be in $\left(R H_{\infty}\right)$ since the function $w(x) \equiv 1$ is.

Conversely, suppose that $\phi$ and $w$ are in $\left(R H_{\infty}\right)$. Fix a cube $I$; then for almost every $x$ in $I$

$$
\phi(x) w(x) \leq C I(\phi) I(w) \leq C I\left(\phi^{2}\right)^{1 / 2} I\left(w^{2}\right)^{1 / 2} .
$$

By Theorem $4.1 \phi$ and $w$ are in $\left(R_{2}\right)$. Therefore, by Theorem 2.6,

$$
I\left(\phi^{2}\right)^{1 / 2} I\left(w^{2}\right)^{1 / 2} \leq C I(\phi w) .
$$

Since the constant is independent of $I, \phi w$ is in $\left(R H_{\infty}\right)$.

As a corollary to Theorem 4.8 we give a new proof of a characterization of the multipliers of $\left(A_{1}\right)$ discovered by Johnson and Neugebauer [15]. To do so we must first characterize the multipliers of $\left(R H_{\infty}\right) \cap\left(A_{p}\right)$.

Lemma 4.9. Fix $p>1$. Then a function $\phi$ is a multiplier of $\left(R H_{\infty}\right) \cap\left(A_{p}\right)$ if and only if $\phi$ is in $\left(R H_{\infty}\right) \cap\left(A_{*}\right)$.

Proof. If $\phi$ is a multiplier, then, arguing as in Theorem 4.8, we see that $\phi^{n}$ is in $\left(A_{p}\right)$ for every positive integer $n$. Therefore, by Theorem $2.2, \phi$ is in $\left(A_{*}\right)$.

Conversely, suppose that $\phi$ is in $\left(R H_{\infty}\right) \cap\left(A_{*}\right)$, and let $w$ be in $\left(R H_{\infty}\right) \cap$ $\left(A_{p}\right)$. Then by Theorem $4.8 \phi w$ is in $\left(R H_{\infty}\right)$, so we only need to show that it is in $\left(A_{p}\right)$. By Theorem 4.4 and the reverse Hölder inequality, $w^{1-p^{\prime}}$ is in $\left(R H_{s}\right) \cap\left(A_{1}\right)$ for some $s>1$. Hence, for every cube $I$,

$$
\begin{aligned}
I(\phi w) I\left((\phi w)^{1-p^{\prime}}\right)^{p-1} & \leq I\left(\phi^{s^{\prime}}\right)^{1 / s^{\prime}} I\left(w^{s}\right)^{1 / s} I\left(\phi^{\left(1-p^{\prime}\right) s^{\prime}}\right)^{(p-1) / s^{\prime}} I\left(w^{\left(1-p^{\prime}\right) s}\right)^{(p-1) / s} \\
& \leq C I(\phi) I\left(\phi^{\left(1-p^{\prime}\right) s^{\prime}}\right)^{(p-1) / s^{\prime}} I(w) I\left(w^{1-p^{\prime}}\right)^{p-1} .
\end{aligned}
$$

Since $\phi$ is in $\left(A_{*}\right)$, it is in $\left(A_{q}\right)$, where $q=(p-1) / s^{\prime}+1$. Therefore the last term in this inequality is bounded for all $I$, so $\phi w$ is in $\left(A_{p}\right)$.

Theorem 4.10. A function $\phi$ is a multiplier of $\left(A_{1}\right)$ if and only if $\phi^{-1}$ is in $\left(R H_{\infty}\right) \cap\left(A_{*}\right)$.

Proof. By Theorem 4.4, a function $w$ is in $\left(A_{1}\right)$ if and only if $w^{-1}$ is in $\left(R H_{\infty}\right) \cap\left(A_{2}\right)$. Hence $\phi$ is a multiplier of $\left(A_{1}\right)$ if and only if $\phi^{-1}$ is a multiplier of $\left(R H_{\infty}\right) \cap\left(A_{2}\right)$. Our conclusion then follows at once from Lemma 4.9.

\section{Applications to the $\left(R H_{S}\right)$ Classes}

In this section we use our results on $\left(R H_{\infty}\right)$ weights to prove two theorems about the reverse Hölder classes, in particular about the class $\left(R H_{s}\right) \cap\left(A_{p}\right)$. The first extends the Jones' factorization theorem for $\left(A_{p}\right)$ weights $[10$, p. 436] to include information about the reverse Hölder classes.

Theorem 5.1. A weight $w$ is in $\left(R H_{s}\right) \cap\left(A_{p}\right), 1 \leq p<\infty, 1<s \leq \infty$, if and only if there exist weights $w_{0}$ and $w_{1}$ such that $w_{0}$ is in $\left(R H_{s}\right) \cap\left(A_{1}\right), w_{1}$ is in $\left(R H_{\infty}\right) \cap\left(A_{p}\right)$, and $w=w_{0} w_{1}$.

Proof. First consider the case when $p=1$ or $s=\infty$. If $w$ is in $\left(A_{p}\right) \cap\left(R H_{s}\right)$, then set either $w_{0}$ or $w_{1}$ equal to one and the other equal to $w$. Conversely, given $w_{0}$ and $w_{1}$, one of them will be in $\left(A_{1}\right) \cap\left(R H_{\infty}\right)$ and so by Corollary 
4.6 will be equivalent to one. It is obvious that multiplying by such a function preserves both $\left(A_{p}\right)$ and $\left(R H_{s}\right)$ classes, so $w_{0} w_{1}$ is in $\left(R H_{s}\right) \cap\left(A_{p}\right)$.

Now suppose that $p>1$ and $s<\infty$. If we are given $w_{0}$ and $w_{1}$, then by Theorem 4.4 there exists a function $v$ in $\left(A_{1}\right)$ such that $w_{1}=v^{1-p}$. So by the Jones' factorization theorem, $w=w_{0} w_{1}=w_{0} v^{1-p}$ is in $\left(A_{p}\right)$. To see that $w$ is in $\left(R H_{s}\right)$, fix a cube $I$. Since $w_{0}$ is in $\left(R H_{\infty}\right)$, there is a constant $C$ such that $w_{0}(x) \leq C I\left(w_{0}\right)$ for almost every $x$ in $I$. Hence

$$
I\left(w_{0}^{s} w_{1}^{s}\right) \leq C I\left(w_{0}^{s}\right) I\left(w_{1}^{s}\right) \leq C I\left(w_{0}\right)^{s} I\left(w_{1}\right)^{s}
$$

the last inequality holds since $w_{0}$ is in $\left(R H_{\infty}\right)$ and so in $\left(R H_{s}\right)$, and since $w_{1}$ is in $\left(R H_{s}\right)$. Finally, since $w_{1}$ is in $\left(A_{1}\right)$, there is a constant $C$ such that $I\left(w_{1}\right) \leq C w_{1}(x)$ for almost every $x$ in $I$. Therefore inequality (10) becomes $I\left(w^{s}\right) \leq C I\left(w_{0} w_{1}\right)^{s}$. Since the constant is independent of $I, w$ is in $\left(R H_{s}\right)$.

Conversely, if $w$ is in $\left(R H_{s}\right) \cap\left(A_{p}\right)$, then by Theorem $2.2, w^{s}$ is in $\left(A_{q}\right)$, where $q=s(p-1)+1$. Therefore, again by the Jones' factorization theorem, there exist $v_{0}, v_{1}$ in $\left(A_{1}\right)$ such that $w^{s}=v_{0} v_{1}^{1-q}$, or equivalently, $w=$ $v_{0}^{1 / s} v_{1}^{1-p}$. Let $w_{0}=v_{0}^{1 / s}$ and $w_{1}=v_{1}^{1-p}$. Since $v_{0}$ is in $\left(A_{1}\right)$, by Theorems 2.1 and $2.3 w_{0}$ is in $\left(R H_{s}\right) \cap\left(A_{1}\right)$; since $v_{1}$ is in $\left(A_{1}\right)$, by Theorem $4.4 w_{1}$ is in $\left(R H_{\infty}\right) \cap\left(A_{p}\right)$.

Theorem 5.1 ties together two central results about $\left(A_{p}\right)$ weights-the reverse Hölder inequality and the Jones' factorization theorem. However, neither of the proofs of these theorems hints at the existence of the other. It would be of interest to have a proof of the latter which contained the additional information about reverse Hölder classes as an integral part of the proof. (Strömberg and Torchinsky [21] include the reverse Hölder class as a hypothesis in their version of the factorization theorem. However, their conclusion is much weaker than ours.)

In our second result we determine the action of the minimal and maximal operators on the class $\left(R H_{s}\right) \cap\left(A_{p}\right)$. Roughly speaking, we show that the $\left(R H_{s}\right)$ class of a function is determined by its behavior on the set where its values are large and its $\left(A_{p}\right)$ class by its behavior on the set where its values are small. This interpretation cannot be taken too literally since it is possible to construct bounded $\left(A_{\infty}\right)$ which are not in $\left(R H_{\infty}\right)$ and $\left(A_{\infty}\right)$ weights which are bounded away from zero which are not in $\left(A_{1}\right)$. (By Corollary 4.6 and the preceding remarks, it suffices to construct a non-positive function $\phi$ in BMO such that $-\phi$ is not in BLO. Also, see Example 5.3 below.) Pari of this result, that $M w$ is in $\left(A_{1}\right)$ whenever $w$ is in $\left(A_{\infty}\right)$, was first discovered by Johnson and Neugebauer [14].

Theorem 5.2. Let $w$ be in $\left(R H_{s}\right) \cap\left(A_{p}\right), 1<s \leq \infty, 1 \leq p<\infty$. Then $M w$ is in $\left(R H_{s}\right) \cap\left(A_{1}\right)$ and $m w$ is in $\left(R H_{\infty}\right) \cap\left(A_{p}\right)$.

Proof. First suppose that $w$ is in $\left(R H_{s}\right) \cap\left(A_{p}\right), s<\infty$ and $p>1$. We first consider $M w$. Since $w$ is in $\left(R H_{s}\right)$, there is a constant $C$ such that for every cube $I, I(w) \leq I\left(w^{s}\right)^{1 / s} \leq C I(w)$. Fix a point $x$ and take the supremum over all cubes $I$ containing $x$. Then

$$
M w(x) \leq M\left(w^{s}\right)(x)^{1 / s} \leq C M w(x) .
$$


Since $1 / s<1$, by Theorem 6.1 below $M\left(w^{s}\right)^{1 / s}$ is in $\left(A_{1}\right)$, so $M w$ is as well. To see that $M w$ is in $\left(R H_{s}\right)$, note that since $w$ is in $\left(R H_{s}\right)$, by Theorem 2.1 $w^{s}$ is in $\left(A_{\infty}\right)$ and so in some reverse Hölder class. Then by the same argument as before we see that $M\left(w^{s}\right)$ is in $\left(A_{1}\right)$. Hence $(M w)^{s}$ is in $\left(A_{1}\right)$, so, again by Theorem $2.1, M w$ is in $\left(R H_{s}\right)$.

Now consider $m w$. Since $w$ is in $\left(A_{p}\right), w^{1-p^{\prime}}$ is in $\left(A_{p^{\prime}}\right)$, so by the above argument $M\left(w^{1-p^{\prime}}\right)$ is in $\left(A_{1}\right)$. Hence by Theorem $4.4, M\left(w^{1-p^{\prime}}\right)^{1-p}$ is in $\left(R H_{\infty}\right) \cap\left(A_{p}\right)$. But then by inequality (8) of Lemma $4.3, m w$ is also in the same class.

Now suppose that $w$ is in $\left(R H_{\infty}\right) \cap\left(A_{p}\right)$. Then $m w \sim w$, so it is immediate that $m w$ is in this class as well. Since $w$ is in $\left(R H_{\infty}\right)$, by Theorem 4.1 it is in $\left(R H_{s}\right)$ for all $s>1$, so by the previous argument $M w$ is in $\left(A_{1}\right) \cap\left(R H_{s}\right)$. Furthermore, a close examination of the proof shows that the $\left(R H_{s}\right)$ constant of $M w$ depends only on the reverse Hölder constant of $w^{s}$, and this constant is independent of $s$ since $w$ is in $\left(R H_{\infty}\right)$. Therefore, again by Theorem 4.1, Mw is in $\left(R H_{\infty}\right) \cap\left(A_{1}\right)$.

Finally, suppose that $w$ is in $\left(A_{1}\right) \cap\left(R H_{s}\right)$. Then $M w \sim w$, so $M w$ is in the same class. By Theorem $4.4 w^{-1}$ is in $\left(R H_{\infty}\right) \cap\left(A_{2}\right)$. Therefore, by the previous argument, $M\left(w^{-1}\right)$ is in $\left(R H_{\infty}\right) \cap\left(A_{1}\right)$ and so, by Corollary 4.6 , is equivalent to one. Hence by inequality (8) of Lemma 4.3, $m w \sim 1$ and so is in $\left(A_{1}\right) \cap\left(R H_{\infty}\right)$.

The conclusion of Theorem 5.2 is sharp: The function $w(t)=|t|^{-1 / 2}$ is in $\left(R H_{s}\right) \cap\left(A_{1}\right)$ for all $s<2$ but not in $\left(R H_{2}\right)$, and $M w$ has the same property. Similarly, let $w(t)=|t|^{1 / 2}$. Then $w$ and $m w$ are in $\left(R H_{\infty}\right) \cap\left(A_{p}\right)$ for all $p>2$ but are not in $\left(A_{2}\right)$.

Theorem 5.2 has only a very weak converse: If $w$ is in $\left(A_{1}\right)$ and $M w$ is in $\left(R H_{s}\right)$, then so is $w$; and if $w$ is in $\left(R H_{\infty}\right)$ and $m w$ is in $\left(A_{p}\right)$, then so is $w$. No stronger converse is possible, as the next example shows. (We would like to thank Don Sarason for showing us an illuminating construction which led to this example.)

Example 5.3. There exists a function $w$ in $\left(A_{\infty}\right)$ such that $M w$ is in $\left(R H_{\infty}\right)$ but $w$ is not in $\left(\mathrm{RH}_{2}\right)$. Similarly, there exists a function $v$ in $\left(A_{\infty}\right)$ such that $m v$ is in $\left(A_{1}\right)$ but $v$ is not in $\left(A_{2}\right)$.

Proof. Both examples will be on $\mathbb{R}_{+}$. We will construct a function $w$ which is bounded, less than or equal to one on $[0,1]$ and equal to one on $(1, \infty)$, and in $\left(A_{\infty}\right)$ but not in $\left(R H_{2}\right)$. But $M w$ will be identically equal to one and so in $\left(R H_{\infty}\right)$.

For each $n \geq 0$, define $f_{n}$ to be the truncation of $|t|^{-1 / 2}$ at height $2^{n / 2}$ :

$$
f_{n}(t)= \begin{cases}|t|^{-1 / 2} & \text { if } 2^{-n} \leq|t| \leq 1 \\ 2^{n / 2} & \text { if } 0 \leq|t| \leq 2^{-n}\end{cases}
$$

Then

$$
\int_{-1}^{1} f_{n}(t) d t \leq 4
$$


Now by scaling and translating $f_{n}(t)$, define the function $g_{n}(t)$ with domain $I_{n}=\left[2^{-(n+1)}, 2^{-n}\right]$. Then

$$
\int_{I_{n}} g_{n}(t) d t \leq 4\left|I_{n}\right|
$$

Lastly, let $w_{n}(t)=2^{-n / 2} g_{n}(t)$ and define

$$
w(t)=\sum_{n=0}^{\infty} w_{n}(t) \chi_{I_{n}}(t), \quad 0<t \leq 1 .
$$

Notice that $w(t) \leq 1$ on $[0,1]$. Extend $w$ to a function on $\mathbb{R}_{+}$by setting it equal to one on $(1, \infty)$. We claim that $w$ is in $\left(A_{\infty}\right)$. Since $w$ is piecewise monotonic, it will suffice to show that $w$ is in $\left(A_{p}\right)$ for some $p$ on small subintervals of $[0,1]$ either contained in one of the $I_{n}$ or adjacent to the origin. (See [4] for details.) If $I$ is contained in some $I_{n}$, then $w$ behaves like $t^{-1 / 2}$, which is in $\left(A_{1}\right)$. If $I$ is adjacent to the origin, then $w$ behaves like $t^{1 / 2}$, which is in $\left(A_{p}\right)$ for $p>3 / 2$.

To see that $w$ is not in $\left(R H_{2}\right)$ : For any $n \geq 0$ we have the estimate

$$
I_{n}(w)^{2}=I_{n}\left(w_{n}\right)^{2} \leq\left(4 \cdot 2^{-n / 2}\right)^{2}=2^{4-n} .
$$

However, $I_{n}\left(w_{n}^{2}\right)=2^{-n}(n \log 2+2)$. Therefore, as $n$ tends to infinity the ratio $I_{n}(w)^{2} / I_{n}\left(w^{2}\right)$ tends to zero, so $w$ cannot be in $\left(R H_{2}\right)$.

Finally, we construct the function $v$ from $w$. The above construction actually showed that $w$ is in $\left(A_{p}\right)$ for $p>3 / 2$ but is not in $\left(A_{3 / 2}\right)$ (since $t^{1 / 2}$ is not). Therefore, by the duality of $\left(A_{p}\right)$ weights, $v=w^{-2}$ is in $\left(A_{p}\right)$ for $p>2$ but is not in $\left(A_{2}\right)$. But it follows from the definition of $w$ that $m v \equiv 1$ and so lies in $\left(A_{1}\right)$.

We conclude this section with a curious corollary to Theorems 5.1 and 5.2:

Corollary 5.4. If $w$ is in $\left(R H_{s}\right) \cap\left(A_{p}\right)$, then so is $M w m w$.

Though $w$ and $M w m w$ both lie in the same $\left(A_{p}\right)$ and $\left(R H_{s}\right)$ classes, they are not generally equivalent. This naturally raises the following question:

Question 5.5. Given a function $w$ in $\left(R H_{s}\right) \cap\left(A_{p}\right)$, when is $w \sim M w m w$ ?

Though we have not made any progress on this question, it is clear that a solution will cast additional light onto the decomposition of a BMO function into the difference of two BLO functions.

\section{The STRUCTURE OF $\left(A_{1}\right)$ AND $\left(R H_{\infty}\right)$ WeIGHTS}

Because of the close relationship between $\left(R H_{\infty}\right)$ weights and $\left(A_{1}\right)$ weights, it seemed reasonable to ask if there is a characterization of $\left(R H_{\infty}\right)$ weights similar to the one for $\left(A_{1}\right)$ weights given by Coifman and Rochberg [8]:

Theorem 6.1. Given a measure $\mu$ on $\mathbb{R}^{n}$, then for all $r, 0<r<1$, the function $(M \mu)^{r}$ is in $\left(A_{1}\right)$. Conversely, if $w$ is in $\left(A_{1}\right)$ there exists a function $v$, and $r, 0<r<1$, such that $w \sim(M v)^{r}$. 
An analogous result for $\left(R H_{\infty}\right)$ would involve functions of the form $(m w)^{r}, 0<r<1$. But by Theorem 4.2, if $(m w)^{r}$ is in $\left(R H_{\infty}\right)$, then so is $m w$. Therefore the problem becomes to characterize those functions $w$ such that $m w$ is in $\left(R H_{\infty}\right)$; the analogous question for $\left(A_{1}\right)$ weights is not answered by Theorem 6.1. These two problems are related, which is not surprising considering the close relation between $\left(A_{1}\right)$ and $\left(R H_{\infty}\right)$ shown above. We give solutions to both problems in the next two theorems. Theorem 6.2 first appeared in [4]; we include the proof here for completeness.

Theorem 6.2. Given a function $w, M w$ is in $\left(A_{1}\right)$ if and only if there exist constants $r>1$ and $C$ such that $M\left(w^{r}\right) \leq C(M w)^{r}$.

Proof. By Theorem 6.1 the function $\left(M w^{r}\right)^{1 / r}$ is in $\left(A_{1}\right)$. But by our hypothesis $M w \sim\left(M w^{r}\right)^{1 / r}$, so $M w$ is also in $\left(A_{1}\right)$.

Conversely, if $M w$ is in $\left(A_{1}\right)$, then it is $\left(R H_{r}\right)$ for some $r>1$. Hence there is a constant $C$ such that for all cubes $I, I\left((M w)^{r}\right)^{1 / r} \leq C I(M w)$. Taking the supremum over $I$ shows that

$$
M\left((M w)^{r}\right)^{1 / r} \leq C M(M w) \leq C M w .
$$

Therefore $M\left(w^{r}\right) \leq M\left((M w)^{r}\right) \leq C(M w)^{r}$.

Using Theorem 6.2 we can answer our original question.

Theorem 6.3. Given a function $w, m w$ is in $\left(R H_{\infty}\right)$ if and only if there exist constants $p, 1<p<\infty$, and $C$ such that

$$
1 \leq m w M\left(w^{1-p^{\prime}}\right)^{p-1} \leq C .
$$

Proof. Suppose first that $m w$ is in $\left(R H_{\infty}\right)$. Then $m w$ is in $\left(A_{p}\right)$ for some $p>1$. By Hölder's inequality the first inequality in (11) always holds, so it will suffice to show the second. By inequality (8) of Lemma 4.3, there exists a constant $C$ such that

$$
m(m w) M\left((m w)^{1-p^{\prime}}\right)^{p-1} \leq C .
$$

Since $m w$ is in $\left(R H_{\infty}\right)$, there exists a constant such that $m w \leq$ $C m(m w)$. Since $w \geq m w$ almost everywhere,

$$
M\left((m w)^{1-p^{\prime}}\right)^{p-1} \geq M\left(w^{1-p^{\prime}}\right)^{p-1} .
$$

These three inequalities combined show that inequality (11) holds.

Conversely, suppose that inequality (11) holds for some $p$. Fix $q>p$. Then $q^{\prime}<p^{\prime}$, so

$$
M\left(w^{1-q^{\prime}}\right)^{q-1} \leq M\left(w^{1-p^{\prime}}\right)^{p-1} \leq \frac{C}{m w} \leq C M\left(w^{1-q^{\prime}}\right)^{q-1},
$$

the first and third inequalities following from Hölder's inequality, and the second from inequality (11). Let $s=\left(p^{\prime}-1\right) /\left(q^{\prime}-1\right)$. Then $s>1$, so $M\left(w^{1-q^{\prime}}\right)$ satisfies the condition given in Theorem 6.2 and thus is in $\left(A_{1}\right)$. Further, $m w \sim M\left(w^{1-q^{\prime}}\right)^{1-q}$, so by Theorem $4.1 \quad m w$ is in $\left(R H_{\infty}\right)$.

Theorems 6.2 and 6.3 may be summarized as saying that $M w$ is in $\left(A_{1}\right)$ when $w$ satisfies a reverse-Hölder-type condition and that $m w$ is in $\left(R H_{\infty}\right)$ 
whenever $w$ satisfies an $\left(A_{p}\right)$-type condition. Thus these results generalize Theorem 5.2. (Theorem 5.2 also gives large classes of functions which satisfy the conditions in these results.)

In fact, the proofs of these two theorems give information about the $\left(R H_{r}\right)$ and $\left(A_{p}\right)$ classes to which $M w$ and $m w$ belong. Clearly, if $M w$ is in $\left(R H_{r}\right)$, then $w$ satisfies $M\left(w^{r}\right) \leq C(M w)^{r}$. Conversely, by Theorem 6.1 if $t<r$, then $\left(M w^{r}\right)^{t / r}$ is in $\left(A_{1}\right)$, so by Theorem $2.1 M w$ is in $\left(R H_{t}\right)$ for all $t<r$. Similarly, if $m w$ is in $\left(A_{p}\right)$, then $w$ satisfies inequality (11); if $w$ satisfies (11), then $m w$ is in $\left(A_{q}\right)$ for all $q>p$. This lack of symmetry led us to the following two conjectures.

Question 6.4. If a function $w$ satisfies $M\left(w^{r}\right) \leq C(M w)^{r}$ for some $r>1$, is $M w$ in $\left(R H_{r}\right)$ ?

Question 6.5. If a function $w$ satisfies $1 \leq m w M\left(w^{1-p^{\prime}}\right)^{p-1} \leq C$ for some $p>1$, is $m w$ in $\left(A_{p}\right)$ ?

The proof of Theorem 6.3 can be easily adapted to show that Question 6.4 implies Question 6.5. However, Question 6.4 is extremely difficult and repeated attempts either to prove it or to construct a counterexample have been unsuccessful. It is easy to see that it is equivalent to the following question.

Question 6.6. If a function $w$ satisfies $M\left(w^{r}\right) \leq C(M w)^{r}$ for some $r>1$, do there exist constants $C^{\prime}$ and $s>r$ such that $M\left(w^{s}\right) \leq C^{\prime}(M w)^{s}$ ?

This reformulation of the question shows that this problem is closely related to a number of so-called higher integrability theorems which are important in the study of partial differential equations and quasi-conformal mappings. Gehring [11] proved that if a function $w$ is supported in a cube $I$ and satisfies the inequality $M\left(w^{r}\right) \leq C(M w)^{r}$ on $I$, then there exists $s>r$ such that $I\left(w^{s}\right) \leq C I(w)^{s}$. However, the statement of his theorem omitted the crucial hypothesis that $w$ is zero off of $I$. Were the theorem true as stated, then Question 6.6 would be a corollary of it. Giaquinta and Modica [13] showed that this hypothesis cannot be omitted, but their counterexample does not apply to Question 6.6. Giaquinta [12] and Stredulinsky [20] independently proved a more general version of Gehring's result which again requires that $w$ be compactly supported. Question 6.6 generalizes Gehring's result in another direction: It omits the hypothesis of compact support but replaces the integral inequality in the conclusion with a weaker one involving maximal functions.

We conclude this section with an example of a large class of functions which satisfy the condition of Theorem 6.2 and for which Question 6.6 is true. We begin with a definition.

Definition 6.7. A function $w$ satisfies the weak reverse Hölder inequality with exponent $r>1$ if there exists a constant $C$ such that for every cube $I$

$$
\left(\frac{1}{|I|} \int_{I} w^{r} d x\right)^{1 / r} \leq \frac{C}{|2 I|} \int_{2 I} w d x
$$

We denote this by saying that $w$ is in weak $\left(R H_{r}\right)$. The union of all the weak reverse Hölder classes is called weak $\left(A_{\infty}\right)$. 
Functions which are in the weak reverse Hölder classes come up naturally in the study of partial differential equations, quasi-conformal mappings and weighted norm inequalities. Sawyer [19] first called the union of all the weak $\left(R H_{r}\right)$ classes weak $\left(A_{\infty}\right)$ and proved that this class has several equivalent characterizations similar to the original $\left(A_{\infty}\right)$ condition.

If a function $w$ is in weak $\left(R H_{r}\right)$, then by an argument essentially the same as the second half of the proof of Theorem 6.2, $M\left(w^{r}\right) \leq C(M w)^{r}$. Hence $M w$ is in $\left(A_{1}\right)$. Furthermore, Giaquinta [12] has shown that if $w$ is in weak $\left(R H_{r}\right)$, then it is in weak $\left(R H_{s}\right)$ for some $s>r$, so $M\left(w^{s}\right) \leq C(M w)^{s}$.

It is not clear whether functions in weak $\left(A_{\infty}\right)$ are examples of functions which satisfy inequality (11) of Theorem 6.3 . The difficulty is that weak $\left(A_{\infty}\right)$ does not appear to have a natural duality-there exist $w$ in weak $\left(A_{\infty}\right)$ such that $w^{-r}$ is not for any $r>0$. For example, let $w(t)=\chi_{[0, \infty)}(t)$.

\section{THE MAXIMAL OPERATOR AND BMO}

In this section we apply our results to give a new proof that the maximal operator is bounded on BMO. This was first proved by Bennett, DeVore, and Sharpley [2]; another proof with some affinity to ours was given by Chiarenza and Frasca [3].

The heart of our proof is the following "commutation" result which we think is of interest in itself.

Theorem 7.1. Let $w=e^{\phi}$ be in $\left(A_{p}\right)$ for some $p>1$. Then $M \phi$ is in $B L O$ and

$$
M \phi-\log (\max (M w, 1 / m w))
$$

is in $L^{\infty}$, and its norm depends only on the $\left(A_{p}\right)$ constant of $w$. If $w$ is in $\left(A_{1}\right)$, then $M \phi-\log M w$ is in $L^{\infty}$ and its norm depends only on the $\left(A_{1}\right)$ constant of $w$. If $w$ is in $\left(R H_{\infty}\right)$, then $M \phi+\log m w$ is in $L^{\infty}$.

Proof. The proof depends on several inequalities satisfied by $\left(A_{p}\right)$ weights. It has two cases depending on the size of $p$.

Suppose first that $p \leq 2$. Then $w$ is in $\left(A_{2}\right)$, so there exists a constant $C$, depending only on the $\left(A_{p}\right)$ constant of $w$, such that for every cube $I$, $I\left(e^{|\phi-I(\phi)|}\right) \leq C[10$, p. 409]. Hence by Jensen's inequality

$$
I(|\phi|) \leq|I(\phi)|+C .
$$

Now again by Jensen's inequality $I(\phi) \leq \log I(w)$. Further, $w$ satisfies the so-called reverse Jensen inequality: There exists a constant $C$, depending only on the $\left(A_{p}\right)$ constant of $w$, such that for every cube $I, I(w) \leq C e^{I(\phi)}[10, \mathrm{p}$. 405]. It follows from this that $-I(\phi) \leq-\log I(w)+C$. Together, these two inequalities show that for any $x$ in $I$

$$
\begin{aligned}
|I(\phi)| & \leq \max (\log I(w),-\log I(w))+C \\
& \leq \max (\log M w(x),-\log m w(x))+C \\
& =\log (\max (M w(x), 1 / m w(x)))+C .
\end{aligned}
$$

Combining this with inequality (14) we get

$$
I(|\phi|) \leq \log (\max (M w(x), 1 / m w(x)))+C .
$$


Therefore, if we fix $x$ and take the supremum over all cubes $I$ which contain it we see that

$$
M \phi(x) \leq \log (\max (M w(x), 1 / m w(x)))+C .
$$

To show that (13) is in $L^{\infty}$ we need to establish the reverse of this inequality. By the reverse Jensen inequality, for every cube $I$ and $x$ in $I, I(w) \leq C e^{I(\phi)} \leq$ $C e^{M \phi(x)}$, so if we again fix $x$ and take the supremum over all $I$ containing it we get

$$
\log M w(x) \leq M \phi(x)+C .
$$

Similarly, by Jensen's inequality, for every $I$ and $x$ in $I,-\log I(w) \leq I(-\phi) \leq$ $M \phi(x)$, so

$$
-\log m w(x) \leq M \phi(x) .
$$

Together, inequalities (16) and (17) imply the reverse of (15), so (13) is in $L^{\infty}$. Furthermore, all of the constants involved in the proof depend only on the $\left(A_{p}\right)$ constant of $w$, so its $L^{\infty}$ norm does as well.

To see that $M \phi$ is in BLO: Since $w$ is in $\left(A_{2}\right)$, by Theorems 4.4 and 5.2 $M w$ and $1 / m w$ are both in $\left(A_{1}\right)$. Then a simple calculation shows that $\max (M w, 1 / m w)$ is also in $\left(A_{1}\right)$. Hence the logarithm of this maximum is in BLO [10, p. 409]. The sum of a bounded function and a function in BLO is again in BLO, so $M \phi$ is in BLO.

Now suppose that $p>2$. Then by the duality of $\left(A_{p}\right)$ weights, $w^{1-p^{\prime}}=$ $e^{\left(1-p^{\prime}\right) \phi}$ is in $\left(A_{p^{\prime}}\right)$. Since $p^{\prime}<2$, by the previous case $M \phi$ is in BLO and

$$
\left(p^{\prime}-1\right) M \phi-\log \left(\max \left(M\left(w^{1-p^{\prime}}\right), 1 / m\left(w^{1-p^{\prime}}\right)\right)\right)
$$

is in $L^{\infty}$. By Lemma 4.3 the second term differs from the function

$$
\left(p^{\prime}-1\right) \log (\max (M w, 1 / m w))
$$

by a bounded function whose $L^{\infty}$ norm depends only on the $\left(A_{p}\right)$ constant of $w$. Therefore (13) is in $L^{\infty}$; since the $\left(A_{p}\right)$ constant of $w$ is the same as the $\left(A_{p^{\prime}}\right)$ constant of $w^{1-p^{\prime}}$, its $L^{\infty}$ norm depends only on the former.

If $w$ is in $\left(A_{1}\right)$ it is in $\left(A_{2}\right)$, so by the first case (13) is in $L^{\infty}$. But by Theorem 5.2 and Corollary $4.6 m w$ is in $\left(R H_{\infty}\right) \cap\left(A_{1}\right)$ and thus bounded and bounded below. Hence $M \phi-\log M w$ is in $L^{\infty}$. Finally, an identical argument shows that if $w$ is in $\left(R H_{\infty}\right)$ then $M w$ is bounded and bounded below and so $M \phi+\log m w$ is in $L^{\infty}$.

Theorem 7.2. The maximal operator is a bounded operator on BMO and maps $B M O$ into $B L O$.

Proof. It is a consequence of the John-Nirenberg theorem that if $\phi$ is in BMO then there exists a constant $\lambda$, depending only on the norm of $\phi$, such that $w=e^{\lambda \phi}$ is in $\left(A_{2}\right)$ and the $\left(A_{2}\right)$ norm of $w$ is bounded by an absolute constant $[10$, pp. 166,409$]$. Since the maximal operator is positive homogeneous, it is immediate from Theorem 7.1 that the maximal operator maps BMO into BLO.

To see that the maximal operator is a bounded operator, fix a constant $K$ such that if $\|\phi\|_{*}<K$, then $w=e^{\phi}$ is in $\left(A_{2}\right)$ and its $\left(A_{2}\right)$ constant is 
independent of $\phi$. Then by Theorem 7.1

$$
M \phi=\log (\max (M w, 1 / m w))+\psi,
$$

where $\psi$ is a bounded function whose $L^{\infty}$ norm, and so whose BMO norm, is independent of $\phi$. By the proof of Theorem 5.2, the $\left(A_{1}\right)$ constants of $M w$ and $1 / m w$ depend only on the $\left(A_{2}\right)$ constant of $w$, so the $\left(A_{1}\right)$ constant of their maximum is independent of $\phi$. But the BMO norm of the logarithm of an $\left(A_{p}\right)$ function depends only on its $\left(A_{p}\right)$ constant; hence the norm of the right-hand side of (18) is a constant independent of $\phi$. Therefore the maximal operator is bounded.

\section{APPENDIX}

This section is an erratum to an earlier paper by the first author [5] which contains two results that are incorrect as stated. The correct theorems are the following.

Theorem 8.1. If $w$ is in $\left(A_{1}\right)$ and $f$ and $|f| \log ^{+}|f|$ are in $L^{1}(w)$ (on the unit circle), then $M f$ is in $L^{1}(w)$. Conversely, if $w$ is in $\left(R H_{\infty}\right)$ and $f$ and $M f$ are in $L^{1}(w)$, then $|f| \log ^{+}|f|$ is in $L^{1}(w)$.

Theorem 8.2. If $w$ is in $\left(A_{1}\right)$ and if $f$ and $|f| \log ^{+}|f|$ are in $L^{1}(w)$ (on the unit circle), then $\tilde{f}$, the conjugate function of $f$, is in $L^{1}(w)$. Conversely, if $w$ is in $\left(R H_{\infty}\right)$ and if $f \geq 0$ and $f, \tilde{f}$ are in $L^{1}(w)$, then $|f| \log ^{+}|f|$ is in $L^{1}(w)$.

The first halves of Theorems 8.1 and 8.2 are correct in the original paper. However, the incorrect condition that $w$ is in $\left(A_{1}\right)$ is assumed for the second half of each theorem. Necessary and sufficient conditions on the weight $w$ for the second half of Theorem 8.1 to hold were given by Andersen and Young [1] and Muckenhoupt [18]. They showed that a sufficient condition on $w$ is for it to be in $\left(R H_{\infty}\right)$. And, as noted in [5], the proof of the second half of Theorem 8.2 only uses the second half of Theorem 8.1 and the fact that $w$ is in $\left(A_{\infty}\right)$, so the the proof still holds for $w$ in $\left(R H_{\infty}\right)$.

\section{REFERENCES}

1. K. F. Andersen and W. S. Young, On the reverse weak type inequality for the Hardy maximal function and the weighted classes $L(\log L)^{k}$, Pacific J. Math. $112(1984), 257-264$.

2. C. Bennett, R. A. DeVore, and R. Sharpley, Weak $L \infty$ and BMO, Ann. of Math. 113 (1981), 601-611.

3. F. Chiarenza and M. Frasca, Morrey spaces and Hardy-Littlewood maximal function, Rend. Mat. Appl. (7) 7 (1981), 273-279.

4. D. Cruz-Uribe, SFO, Piecewise monotonic doubling measures, Rocky Mountain J. Math. (to appear).

5. Contemp. Math., vol. 137, Amer. Math. Soc., Providence, RI, 1992.

6. D. Cruz-Uribe, SFO, C. J. Neugebauer and V. Olesen, Norm inequalities for the minimal and maximal operator, and differentiation of the integral, preprint.

7. R. Coifman and C. Fefferman, Weighted norm inequalities for maximal functions and singular integrals, Studia Math. 51 (1974), 241-250. 
8. R. Coifman and R. Rochberg, Another characterization of BMO, Proc. Amer. Math. Soc. 79 (1980), 249-254.

9. B. Franchi, Weighted Sobolev-Poincare inequalities and pointwise estimates for a class of degenerate elliptic equations, Trans. Amer. Math. Soc. 327 (1991), 125-158.

10. J. Garcia-Cuerva and J. L. Rubio de Francia, Weighted norm inequalities and related topics, North-Holland Math. Stud., vol. 116, North-Holland, Amsterdam, 1985.

11. F. W. Gehring, The $L^{p}$-integrability of the partial derivatives of a quasiconformal mapping, Acta Math. 130 (1973), 265-277.

12. M. Giaquinta, Multiple integrals in the calculus of variations and nonlinear elliptic systems, Ann.of Math. Stud., no. 105, Princeton University Press, Princeton, NJ, 1983.

13. M. Giaquinta and G. Modica, Regularity results for some classes of higher order nonlinear elliptic systems, J. Reine Angew. Math. 311/312 (1979), 145-169.

14. R. Johnson, Changes of variable and $\left(A_{p}\right)$ weights, Harmonic Analysis and Partial Differential Equations, Contemp. Math., vol. 107, Amer. Math. Soc., Providence, RI, 1990, pp. 93-99.

15. R. Johnson and C. J. Neugebauer, Homeomorphisms preserving $\left(A_{p}\right)$, Rev. Mat. Iberoamericana 3 (1987), 249-273.

16. $\longrightarrow$, Change of variable results for $\left(A_{p}\right)$-and reverse Hölder $R_{H}{ }_{r}$-classes, Trans. Amer. Math. Soc. 328 (1991), 639-666.

17. J. L. Journé, Zygmund operators, pseudo-differential operators and the Cauchy integral of Calderon, Lecture Notes in Math., vol. 994, Springer-Verlag, New York, 1983.

18. B. Muckenhoupt, Weighted reverse weak type inequalities for the Hardy-Littlewood maximal function, Pacific J. Math. 117 (1985), 371-377.

19. E. Sawyer, Two weight norm inequalities for certain maximal and integral operators, Lecture Notes in Math., vol. 908, Springer-Verlag, New York, 1982, pp. 102-127.

20. E. Stredulinsky, Weighted inequalities and degenerate elliptic partial differential equations, Lecture Notes in Math., vol. 1074, Springer-Verlag, Berlin, 1984.

21. J. O. Strömberg and A. Torchinsky, Weighted Hardy spaces, Lecture Notes in Math., vol. 1381, Springer-Verlag, Berlin, 1989.

22. J. O. Strömberg and R. L. Wheeden, Fractional integrals on weighted $H^{p}$ and $L^{p}$ spaces, Trans. Amer. Math. Soc. 287 (1985), 293-321.

Department of Mathematics, Purdue University, West Lafayette, Indiana 47907-1395

E-mail address: cruz@math.purdue.edu

$E$-mail address: neug@math.purdue.edu 